\title{
Interspecies differences in metabolism of deoxypodophyllotoxin in hepatic microsomes from human, monkey, rat, mouse and dog
}

Qiushi Xie, Yang Chen, Fei Liu, Zeyu Zhong, Kaijing Zhao, Zhaoli Ling, Fan Wang, Xiange Tang, Zhongjian Wang, Li Liu*, Xiaodong Liu*

\author{
Affiliation: \\ Center of Drug Metabolism and Pharmacokinetics, China Pharmaceutical University, \\ Nanjing, 210009, China
}

\section{*Corresponding Author:}

E-mail: liulee@yeah.net (L. Liu); xdliu@cpu.edu.cn (X. D. Liu)

Tel: +86-25-83271006; Fax: +86-25-83271060

\section{Abstract}

Deoxypodophyllotoxin (DPT) is a natural lignan product which has drawn much attention due to its pharmacological properties including antitumor effect. The purpose of this study was to investigate interspecies differences in metabolism of DPT in hepatic microsomes from human (HLM), cynomolgus monkey (CyLM), rat (RLM), mouse (MLM) and dog (DLM). Incubation of DPT with hepatic microsomes from five species in the presence of NADPH resulted in formation of seven 
metabolites, five of which were compared with the synthetic standards. M2 was the most abundant metabolite in microsomes from all species. Rank order of intrinsic clearance for M2 formation was RLM $>$ CyLM $>$ MLM $>$ HLM $>$ DLM. In HLM, sulfaphenazole showed the strongest inhibition effect on M2 formation, but neither ticlopidine nor ketoconazole inhibited M2 formation in HLM. Results from cDNA-expressed human CYP450s experiments showed that clearance of M2 formation was much higher in CYP2C9 and CYP2C19 than that in CYP3A4. Contributions of the three CYP450 isoforms to M2 formation in HLM were estimated using relative activity factor (RAF) method or correction by amount of CYP450 isoforms in HLM. M2 formation in HLM was mainly attributed to CYP2C9, followed by CYP2C19. Involvement of CYP3A4 was minor.

Keywords: Deoxypodophyllotoxin; Metabolism; Hepatic microsomes; Cytochrome P450s; Interspecies differences

\section{Introduction}

Deoxypodophyllotoxin (DPT) is a natural lignan product isolated from Chinese medicine Anthriscus sylvestris, Sinopodophyllum emodi (Wall) and Dysosma versipellis (Wall) [1, 2]. It was reported [3] that DPT possessed anti-proliferative, antitumor, antiviral, anti-inflammatory, anti-platelet aggregation and anti-allergic pharmacological properties, among which the antitumor effect has drawn most 
attention. Several studies have demonstrated that DPT belongs to a cytotoxic agent [4-6]. Some derivatives of DPT such as etoposide and teniposide are widely used as effective antineoplastic drugs. DPT is being developed as a potential chemotherapeutic agent in China.

Our previous study [7] showed that DPT was rapidly eliminated in rats with a half life of 70-100 min. Lee SK et al [8] characterized in vitro metabolism of DPT in both human and rat liver microsomes. Their reports showed that DPT was mainly metabolized to seven metabolites (M1-7) (O-dealkylated or hydroxylated) in hepatic microsomes from rat (Fig. 1), but in human hepatic microsomes only five metabolites (M1, M2, M5/M6 and M7) were detected. CYP2C19 and CYP3A4 were believed to be responsible for the metabolism of DPT in HLM. M7 formation from DPT was reported to be mainly catalyzed by CYP3A4 $[9,10]$.

The U.S. Food and Drug Administration (FDA) encourages the identification of differences in drug metabolism between animals used in nonclinical safety assessments and humans as early as possible during the drug development process. In vitro studies using hepatocytes or subcellular fractions of preclinical species and humans often provide valuable information on the metabolic pathways in vivo. Monkey, rat, mouse and dog are common experimental animals in pre-clinical studies of drug discovery and development. The illustration of metabolic pathways in vitro provides guidance to elucidate potential toxicities [11] and to ensure that the selected animal species for long-term safety assessment are exposed to all major metabolites in humans. 
The aim of this study was to investigate both quantitative and qualitative metabolism profiles of DPT in hepatic microsomes from human, cynomolgus monkey, rat, mouse and beagle dog using LC-MS/MS and some of the metabolites were confirmed by synthetic standards. Interspecies kinetics of major metabolite formation was also characterized. cDNA-expressed human CYP450s were further used to determine the CYP450 isoforms responsible for the formation of major metabolites.

\section{Materials and methods}

\subsection{Chemicals and reagents}

DPT (purity $\geq 98.0 \%$ ) and its metabolites (M1, M2, M3, M5 and M7) provided by Medicinal and Chemical Institute of China Pharmaceutical University (Nanjing, China). Diazepam, diclofenac sodium, omeprazole sodium and ticlopidine from the National Institute for the Control of Pharmaceutical and Biological Products (Beijing, China). Sulfaphenazole, D-glucose-6-phosphate, glucose-6-phosphate dehydrogenase and $\beta$-NADP ${ }^{+}$from Sigma-Aldrich (St Louis, MO, USA). Ketoconazole from J\&K Scientific Ltd. (Beijing, China). 5-Hydroxyomeprazole, 4'-hydroxydiclofenac and 6 $\beta$-hydroxytestosterone from Tokyo Chemical Industry (Tokyo, Japan). Other reagents and solvents were of analytical grade or higher available.

\subsection{Microsomes and enzymes}


Liver microsomes from male Mongolia human (HLM, Lot. SUBK), male cynomolgus monkey (CyLM, Lot. ZDD), male ICR/CD-1 mouse (MLM, Lot. STOM) and male beagle dog (DLM, Lot. UKHU) and cDNA-expressed recombinant human CYP450 isoforms (CYP2C9, CYP2C19 and CYP3A4) expressed in E.coli were purchased from Research Institute for Liver Diseases (Shanghai, China). All recombinant enzymes were coexpressed with human CYP-reductase and supplemented with purified human cytochrome b5. .

Hepatic microsomes of rats (RLM) were prepared in our lab. Male Sprague-Dawley rats $(\mathrm{n}=10)$, weighing $220 \pm 20 \mathrm{~g}$ were obtained from Sino-British SIPPR/BK Lab Animal Ltd (Shanghai, China). Hepatic microsomes were prepared by differential ultracentrifugation as described previously [12]. The microsomal pellets were suspended in phosphate-buffered saline $(\mathrm{pH} 7.4)$ containing $20 \%$ glycerin and the protein concentration was determined by BCA Protein Assay Kit (Beyotime Institute of Biotechnology, Nanjing, China). All microsomal samples were pooled and stored at $-80^{\circ} \mathrm{C}$ until use. The animal experiments were approved by the Animal Ethics Committee of China Pharmaceutical University.

\subsection{Metabolism of DPT in hepatic microsomes from five species}

Metabolites of DPT in hepatic microsomes from five species were identified. The microsome incubation mixture (a final volume of $200 \mu \mathrm{l}$ ) consisted of the 
NADPH-generating system $\left(0.5 \mathrm{mM} \beta\right.$-NADP ${ }^{+}, 10 \mathrm{mM}$ D-glucose-6-phosphate, 1 $\mathrm{U} / \mathrm{mL}$ glucose-6-phosphate dehydrogenase and $\left.5 \mathrm{mM} \mathrm{MgCl}_{2}\right), 2.5 \mu \mathrm{M}$ of DPT and hepatic microsomes. Final concentration of DPT was referred to DPT levels in rat plasma [7]. Based on preliminary experiment, levels of the microsome protein were set to be $0.2 \mathrm{mg} / \mathrm{mL}$ for HLM and CyLM, $0.05 \mathrm{mg} / \mathrm{mL}$ for RLM and MLM, and $1 \mathrm{mg} / \mathrm{mL}$ for DLM. The reaction was started by adding NADPH-generating system after pre-incubation at $37^{\circ} \mathrm{C}$ for $5 \mathrm{~min}$ and was terminated by adding $1 \mathrm{~mL}$ ice-cold ethyl acetate after incubation for $30 \mathrm{~min}$. Control incubations were performed without NADPH-generating system. All incubations were carried out in triplicate with organic solvent content less than $1 \%$. DPT and its metabolites were identified by LC-MS/MS, some of which were verified by their standards.

Then, formation of DPT metabolites and depletion of DPT in hepatic microsomes from five species along with incubation time were also measured after incubation for $0,5,10,15,30,45$ and $60 \mathrm{~min}$, respectively.

Biotransformation form M2 and M7 to M1 in hepatic microsomes from five species were also documented. Briefly, M2 $(2 \mu \mathrm{M})$ and M7 $(0.5 \mu \mathrm{M})$ were incubated with hepatic microsomes systems described above for $60 \mathrm{~min}$, respectively. M1 formation was measured using LC-MS/MS.

\subsection{Kinetics of M2 formation in hepatic microsomes from five species}

As M2 was major metabolite of DPT in hepatic microsomes from five species, kinetics of M2 formation was characterized. The incubation conditions were 
optimized to ensure the formation rates were in the linear range and the depletion of DPT was below $20 \%$. A series of DPT concentrations $(0.0196-12.57 \mu \mathrm{M})$ were used. Protein levels of HLM, CyLM, RLM, MLM, and DLM were set to be 0.04, 0.02, $0.002,0.02$ and $0.4 \mathrm{mg} / \mathrm{mL}$, respectively. The incubation time was set to be $5 \mathrm{~min}$.

\subsection{Effects of selective chemical inhibitors on M2 formation in HLM}

M2 formation from DPT in HLM was reported to be mediated by CYP2C family and CYP3A4 [8]. Three selective inhibitors (sulfaphenazole, ticlopidine and ketoconazole) were employed to investigate the contribution of specific enzyme(s) to M2 formation. The concentrations of inhibitors were as follows [13, 14]: sulfaphenazole $(5 \mu \mathrm{M}, 10 \mu \mathrm{M}$ and $40 \mu \mathrm{M})$ for CYP2C9 and ticlopidine $(5 \mu \mathrm{M}, 10 \mu \mathrm{M}$ and $40 \mu \mathrm{M})$ for CYP2C19 and ketoconazole $(0.5 \mu \mathrm{M}, 1 \mu \mathrm{M}$ and $5 \mu \mathrm{M})$ for CYP3A4. The concentration of DPT was $0.156 \mu \mathrm{M}$ (below $\mathrm{K}_{\mathrm{m}}$ value) and microsome protein level was selected to be $0.1 \mathrm{mg} / \mathrm{mL}$ according to preliminary data. Incubation time was set to be $10 \mathrm{~min}$.

2.6 DPT metabolism by recombinant human CYP450 isoforms

Three cDNA-expressed human CYP450 isoforms (CYP2C9, CYP2C19 and CYP3A4) co-expressing cytochrome reductase and cytochrome b5 were used to 
investigate the contribution of CYP450 isoforms on DPT metabolism. DPT (final concentration of $2.5 \mu \mathrm{M}$ ) was incubated with $20 \mathrm{pmol} / \mathrm{mL}$ of CYP450 isoforms for 30 min and metabolites were detected by LC-MS/MS.

Kinetics of M2 formation by CYP2C9, CYP2C19 and CYP3A4 was characterized. A series of concentrations of DPT (0.0196-2.51 $\mu \mathrm{M}$ for CYP2C9, 0.157-12.56 $\mu \mathrm{M}$ for CYP2C19 and 0.0785-5.025 $\mu \mathrm{M}$ for CYP3A4) were incubated with recombinant human CYP450 isoforms for $5 \mathrm{~min}$. CYP450 concentrations were set to be 0.5 pmol/mL for CYP2C9 and CYP2C19 and $20 \mathrm{pmol} / \mathrm{mL}$ for CYP3A4.

\subsection{DPT metabolism by recombinant human CYP450 isoforms}

The contributions of the three CYP450 isoforms to M2 formation were estimated using two different prediction methods decribed previously [15].

Method 1. The percent contributions of CYP2C9, CYP2C19 and CYP3A4 in HLM to M2 formation were estimated based on the contents of the corresponding CYP isoforms in HLM as follows.

$$
\mathrm{CL}_{\mathrm{CYP} 450 \mathrm{~s}}=\mathrm{A}_{\mathrm{s}} \times \mathrm{CL}_{\text {rec-CYP450s }}
$$

Contributions of CYP450s $(\%)=\left(\mathrm{CL}_{\mathrm{CYP} 450 \mathrm{~s}} / \mathrm{CL}_{\mathrm{HLM}}\right) \times 100$

Where $\mathrm{CL}_{\mathrm{CYP} 450 \mathrm{~s}}, \mathrm{~A}_{\mathrm{s}}$ and $\mathrm{CL}_{\mathrm{HLM}}$ were intrinsic clearance of $\mathrm{M} 2$ formation via specific CYP450 isoform in HLM, the content of the specific CYP450 isoform in HLM and total intrinsic clearance of M2 formation in HLM, respectively. The $\mathrm{CL}_{\text {rec-CYP450s }}$ was the measured clerance of M2 formation by the recombinant CYP450 
isoforms. The contents of the three CYP450 isoforms in HLM were cited from Liu X et al's report [16].

Method 2. The percent contributions of CYP2C9, CYP2C19 and CYP3A4 to M2 formation in HLM were also estimated using relative activity factor (RAF) method. $\mathrm{CL}_{\mathrm{CYP} 450 \mathrm{~s}}$ in HLM via the specific CYP450 isoform was estimated using equation 3.

$$
\mathrm{CL}_{\mathrm{CYP} 450 \mathrm{~s}}=\mathrm{RAF}_{\mathrm{CYP} 450 \mathrm{~s}} \times \mathrm{CL}_{\mathrm{rec}-\mathrm{CYP} 450 \mathrm{~s}}
$$

Contributions of CYP450s (\%) to M2 formation was calculated using equation 2. $\mathrm{CL}_{\mathrm{CYP} 450 \mathrm{~s}}$ and $\mathrm{CL}_{\text {rec-CYP450s }}$ are defined above. $\mathrm{RAF}_{\mathrm{CYP} 2 \mathrm{C} 9}, \mathrm{RAF}_{\mathrm{CYP} 2 \mathrm{C} 19}$ and $\mathrm{RAF}_{\mathrm{CYP} 3 \mathrm{~A} 4}$ were measured using the ratio of diclofenac 4'-hydroxylation activity in HLM to that in recombinant CYP2C9, the ratio of omeprazole 5-hydroxylation activity HLM to that in recombinant $\mathrm{CYP} 2 \mathrm{C} 19$ and the ratio of testosterone $6 \beta$-hydroxylation activity in HLM to that in recombinant CYP3A4, respectively. Diclofenac (4 $\mu \mathrm{M})$, omeprazole $(15 \mu \mathrm{M})$ and testosterone $(50 \mu \mathrm{M})$ were incubated with HLM (levels of protein: $0.1 \mathrm{mg} / \mathrm{ml}, 0.3 \mathrm{mg} / \mathrm{ml}$ and $0.1 \mathrm{mg} / \mathrm{ml}$ ) or corresponding recombinant CYP450 isoforms (levels of specific CYP450 isoforms: $1 \mathrm{pmol} / \mathrm{mL}, 5 \mathrm{pmol} / \mathrm{mL}$ and 2 $\mathrm{pmol} / \mathrm{mL}$ ) for $20 \mathrm{~min}$, respectively. 4'-hydroxydiclofenac 5-hydroxyomeprazole and $6 \beta$-hydroxytestosterone in incubation mixtures were measured using HPLC methods described previously with modification [17-19].

\subsection{Drug Assays}


DPT metabolites were identified using LC-MS/MS on Shimadzu MS-8030 system (Shimadzu, Kyoto, Japan) and further confirmed using synthetic standards. Concentrations of DPT and its metabolites (M1, M2 and M7) in microsome mixtures were measured. Diazepam in a volume of $10 \mu \mathrm{L}(200 \mathrm{ng} / \mathrm{mL}$, internal standard $)$ and ethyl acetate $(1 \mathrm{~mL})$ was added to reaction mixture. After shaking for $10 \mathrm{~min}$, the mixture was subsequently centrifuged at $8000 \times g$ for $10 \mathrm{~min}$. The organic layer $(0.85$ $\mathrm{mL}$ ) was transferred and evaporated to dryness in a vacuum evaporator (Thermo, Waltham, MA, USA). The residue was reconstituted in $100 \mu \mathrm{L}$ of methanol-water mixture (volume 1:1). An aliquot of $5 \mu \mathrm{L}$ was injected into the LC-MS/MS system. Chromatographic separation was performed at $40{ }^{\circ} \mathrm{C}$ using a Shim-pack VP-ODS analytical column $(2.0 \mathrm{~mm} \times 150 \mathrm{~mm}, 4.6 \mu \mathrm{m}$, Shimadzu, Kyoto, Japan). The mobile phase was comprised of acetonitrile (phase A) and deionized water containing $0.1 \%$ formic acid (phase B). A binary gradient elution was performed with the following content of phase A. For metabolism identification: initialized at $40 \%$ for $5.5 \mathrm{~min}$, linear gradient $40-60 \%$ from 5.5 to $8.5 \mathrm{~min}$, isocratic $60 \%$ from 8.5 to $9.5 \mathrm{~min}$, then quickly returned to initial and maintained until $13 \mathrm{~min}$ at a flow rate of $0.25 \mathrm{~mL} / \mathrm{min}$. For quantitative analysis of DPT, M1, M2 and M7: initialized at 20\% of, linear gradient $20-80 \%$ A from 0 to $3 \mathrm{~min}$, returned to $20 \%$ of from 3 to $6 \mathrm{~min}$ and maintained there until $9 \mathrm{~min}$ at a flow rate of $0.3 \mathrm{~mL} / \mathrm{min}$. The ions monitored were $[\mathrm{M}+\mathrm{H}]^{+} \mathrm{m} / \mathrm{z} 399.05$ to 231.40 for DPT, $[\mathrm{M}+\mathrm{H}]^{+} \mathrm{m} / \mathrm{z} 403.10$ to 173.40 for $\mathrm{M} 1$, $[\mathrm{M}+\mathrm{H}]^{+} \mathrm{m} / \mathrm{z} 387.10$ to 219.35 for $\mathrm{M} 2,[\mathrm{M}+\mathrm{H}]^{+} \mathrm{m} / \mathrm{z} 415.10$ to 185.40 for $\mathrm{M} 7,[\mathrm{M}+\mathrm{H}]^{+}$ $\mathrm{m} / \mathrm{z} 401.00$ to 185.05 for $\mathrm{M} 3 / 4,[\mathrm{M}+\mathrm{H}]^{+} \mathrm{m} / \mathrm{z} 385.00$ to 231.00 for $\mathrm{M} 5 / 6$ and $[\mathrm{M}+\mathrm{H}]^{+}$ 
$\mathrm{m} / \mathrm{z} 285.05$ to 154.20 for diazepam. The recovery was larger than $80 \%$ and the relative standard deviations of intra-batch and inter-batch were lower than $15 \%$. The linear range of analytes in the samples was $1.9-1000 \mathrm{ng} / \mathrm{mL}$.

\subsection{Data analysis}

Kinetic parameters of M2 formation were estimated using nonlinear regression analysis by Phoenix Winnonlin 6.3 (Pharsight, Mountain View, CA, USA). Eadie-Hofstee plots were used to check for kinetic characteristics.

Apparent $\mathrm{K}_{\mathrm{m}}$ and $\mathrm{V}_{\max }$ values were calculated from Michaelis-Menten equation (eq. 1) or extended Michaelis-Menten equation for two saturable components (eq. 2) [20] Equation was selected according to Eadie-Hofstee plot and Akaike information

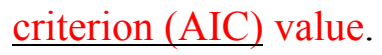

$$
V=\mathrm{V}_{\max } \times S /\left(\mathrm{K}_{\mathrm{m}}+S\right)
$$

Where $V$ is the velocity of M2 formation, $S$ is the concentration of DPT in the incubation mixture, $V_{\max }$ is the maximum metabolic velocity and $\mathrm{K}_{\mathrm{m}}$ is the Michaelis-Menten constant. The intrinsic clearance $\left(\mathrm{CL}_{\text {int }}\right)$ was defined as $\mathrm{V}_{\max } / \mathrm{K}_{\mathrm{m}} \cdot V=$ $\mathrm{V}_{\max 1} \times S /\left(\mathrm{K}_{\mathrm{m} 1}+S\right)+\mathrm{V}_{\max 2} \times S /\left(\mathrm{K}_{\mathrm{m} 2}+S\right)$

Where the $\mathrm{K}_{\mathrm{m} 1}$ and $\mathrm{V}_{\max 1}$ are the Michaelis constant and the maximum velocity of M2 formation for the high affinity component, $K_{m 2}$ and $V_{\max 2}$ are for low affinity component, respectively. The total intrinsic clearance $\left(\mathrm{CL}_{\text {int }}\right)$ was defined as $\mathrm{V}_{\max 1} /$ $\mathrm{K}_{\mathrm{m} 1}+\mathrm{V}_{\max 2} / \mathrm{K}_{\mathrm{m} 2}$.

\section{Results}




\subsection{Metabolism of DPT in hepatic microsomes from five species}

Metabolites of DPT following incubation with hepatic microsomes were identified. It was found that following an incubation time of 30 min with hepatic microsomes from five species, seven metabolites of DPT including M1, M2, M3/4, M5/6 and M7

were detected, five of which were confirmed using corresponding standards (Fig. 2). M2 was the most abundant metabolite among seven metabolites, followed by M1 and M7 while M3/4 and M5/6 were minor ones. In CyLM and DLM, a minor new peak was observed with retention time of $5.5 \mathrm{~min}$, of which the representative fragment 385,231 and 187 were detected with a collision voltage of $15 \mathrm{~V}$, which was in line with those of M5 standard, indicating that it was also an O-dealkylated metabolite of DPT.

Concentrations of M1, M2, M7 and DPT in microsome incubation mixtures following different incubation times were measured (Fig. 3). The results showed that the formation of M1, M2 and M7 gradually increased with incubation time, accompanied by decrease in DPT. Except for DLM, the amount of M7 gradually reduced from 45 to $60 \mathrm{~min}$, accompanied by rapid increase in the amount of M1, suggesting that M7 may be an intermediate product which was further metabolized to M1.

The amount of DPT metabolites in hepatic microsomes from five species was measured following an incubation time of $60 \mathrm{~min}$. Large differences in formation of DPT metabolites were observed among species. $88.3 \%$ of DPT was metabolized to 
M2 in HLM, followed by $67.5 \%$ in MLM, 65.0\% in RLM and 63.0\% in CyLM. But in DLM only $33.5 \%$ of DPT was metabolized to M2. Amounts of M7 and M1 in liver microsomes from the five species were less than that of $\mathrm{M} 2$, but larger interspecies differences was still found. M1 formation in HLM was only $2.12 \%$, which was near to that in MLM (1.90\%), and was markedly lower than those in CyLM (22.84\%), RLM (11.66\%) and DLM (6.90\%). High amount of M7 was also found in DLM (18.62\%) and CyLM (12.30\%), but amounts of M7 in RLM, MLM and HLM were only 5.52\%, $5.20 \%$, and $4.93 \%$, respectivly. M1 formation from M7 and M2 was also documented following 60-min incubation with $\mathrm{M} 2(2 \mu \mathrm{M})$ and $\mathrm{M} 7(0.5 \mu \mathrm{M})$, respectively. The results showed that $13 \%, 11 \%$ and $6.5 \%$ of M2 in RLM, CyLM and DLM were metabolized to M1, respectively. The M1 formation from M2 was less than $1 \%$ in HLM and MLM. However, almost all M7 In RLM, CyLM and HLM was biotransformed to M1, and about $50 \%$ of M7 in MLM and DLM was meatoblized to M1. Summed formation of M1 and M7 (M1+M7) was calculated. Summed formation of M1 and M7 (M1+M7) in HLM was only 7.05\%, which was near to that in MLM (M1+M7: 7.10\%), but markedly lower than that in CyLM (M1+M7: 35.14\%), RLM (M1+M7: 17.18\%) and DLM (M1+M7: 25.52\%).

\subsection{Kinetics of M2 formation in hepatic microsomes from five species}

Kinetics of M2 formation in hepatic microsomes from five species was characterized. As demonstrated by Eadie-Hofstee plots, formation of M2 in HLM, RLM and MLM exhibited Michaelis-Menten kinetics (Fig. 4A, C and D) while the 
kinetics of M2 formation in CyLM and DLM obeyed biphasic kinetics (Fig. 4B and E), with lower AIC values (-103.5 for extended M-M equation versus -65.7 for M-M equation in CyLM; -47.5 for extended M-M equation versus -24.1 for M-M equation in DLM). The apparent kinetic parameters including $\mathrm{K}_{\mathrm{m}}, \mathrm{V}_{\max }$ and $\mathrm{CL}_{\text {int }}$ were estimated (Table 1). The results demonstrated that although two components were involved in M2 formation in CyLM and DLM, the intrinsic clearance of the low affinity component $\left(\mathrm{CL}_{\mathrm{int} 2}=\mathrm{V}_{\max 2} / \mathrm{K}_{\mathrm{m} 2}\right)$ was less than one tenth of the high affinity component $\left(\mathrm{CL}_{\text {int1 }}=\mathrm{V}_{\max 1} / \mathrm{K}_{\mathrm{m} 1}\right)$. RLM exhibited the highest affinity (lowest $\left.\mathrm{K}_{\mathrm{m}}\right)$ and the highest capacity (highest $\mathrm{V}_{\max }$ ), leading to the highest $\mathrm{CL}_{\text {int }}$ of $\mathrm{M} 2$ formation. The rank order of intrinsic clearance for M2 formation was RLM $>$ CyLM $>$ MLM $>$ HLM $>$ DLM.

\subsection{Effects of chemical inhibitors on M2 formation in HLM}

Three chemical inhibitors were used to screen contributions of these CYP450s to M2 formation in HLM (Fig. 5). Sulfaphenazole (CYP2C9 inhibitor) markedly inhibited formation of M2 in a concentration-dependent manner in HLM. Formation of $\mathrm{M} 2$ in presence of $5 \mu \mathrm{M}$ sulfaphenazole was only $43.1 \%$ of control group. However, ticlopidine (CYP2C19 inhibitor) and ketoconazole (CYP3A4 inhibitor) showed little inhibition on M2 formation in HLM, indicating that CYP2C9 was the main CYP isoform responsible for the formation of M2 in HLM.

\subsection{Metabolism of DPT by recombinant human CYP450 isoforms}


Recombinant human CYP2C9, CYP2C19 and CYP3A4 were used to further investigate roles of the three CYP450 isoforms in M2, M1 and M7 formation. Following an incubation time of $30 \mathrm{~min}$ with $20 \mathrm{pmol} / \mathrm{mL}$ of recombinant human CYP2C9 and CYP2C19, large amount of M2, trace amount of M1 and no M7 were observed. In contrast, CYP3A4 was mainly responsible for the formation of M1 and M7 and the formation of M2 mediated by CYP3A4 was less than one fifteenth of CYP2C9 and CYP2C19 (Fig. 6A). Kinetics of M2 formation mediated by CYP2C9, CYP2C19 and CYP3A4 (Fig. 6B, C and D) was investigated. The results showed that although CYP2C9 possessed lower maximum velocity than CYP2C19 did, CYP2C9 showed higher affinity to DPT, leading to higher $\mathrm{CL}_{\text {int }}(0.098 \mathrm{ml} / \mathrm{min} / \mathrm{pmol}$ versus $0.069 \mathrm{ml} / \mathrm{min} / \mathrm{pmol}$ ). The $\mathrm{CL}_{\mathrm{int}}$ of M2 formation by CYP3A4 was $0.002 \mathrm{ml} / \mathrm{min} / \mathrm{pmol}$, which was only one-fiftieth of CYP2C9.

\subsection{Contributions of CYP2C9, CYP2C19 and CYP3A4 to M2 formation in HLM}

Contributions of the CYP450 isoforms to M2 formation in HLM were estimated using two methods (Table 2). The results showed that the contribution of CYP2C9 to M2 formation was the greatest, followed by CYP2C19. The contribution of CYP3A4 to M2 formation was minor. Percent contributions of CYP2C9, CYP2C19 and CYP3A4 to M2 formation in HLM were calculated to be $65.66 \%, 14.61 \%$ and $5.60 \%$, 
respectively, using Method 1. Similar order was obtained using Method 2 although the estimated percent contribution of CYP2C9 to M2 formation was over 100\%.

\section{Discussion}

The present study was undertaken to compare the DPT metabolism profiles in hepatic microsomes from five species and identify the involved enzymes in HLM. The main finding was that the metabolic pathways of DPT were similar in microsomes from five species, in which at least seven metabolites were identified. M2 was the major metabolite. Data from cDNA-expressed CYP450s showed that M2 formation in HLM was mainly catalyzed by CYP2C9 and CYP2C19, with minor involvement of CYP3A4.

In vitro incubations with hepatic systems highlighted the fact that marked species differences in the metabolism of DPT were observed. Following incubation with 2.5 $\mu \mathrm{M}$ of DPT for $60 \mathrm{~min}, 88.3 \%$ of DPT in HLM was metabolized to M2, followed by MLM, RLM and CyLM. In DLM, only $33.5 \%$ of DPT was metabolized to M2. Although M2 and M7 could both be further biotransformed to M1 in microsomes from all species, in vitro incubation showed that M1 formation from M2 was of less significance for total amount of M2 while biotransformation from M7 to M1 was noticeable. Higher velocity of M1 formation from M2 and M7 in RLM and CyLM may contribute to the larger proportion of M1. Metabolism of DPT via M1 and M7 pathways in HLM was only $7.05 \%$, which was near to MLM, but markedly lower than that of other tested animals. These results indicated that M1 and M7 formation 
was also important metabolic pathway of DPT in RLM, DLM, and CyLM. Almost all of DPT related mass was found in HLM and CyLM while 10\%-20\% of DPT related mass still needed to be identified in RLM, MLM and DLM.

Kinetics of M2 formation was characterized. Although biphasic kinetics was observed in DLM and CyLM, intrinsic clearance of M2 formation for low affinity component was less than one tenth of high affinity component, inferring that contribution of low affinity component to M2 formation in CyLM and DLM may be negligible. Large species differences in the intrinsic clearance of M2 formation were observed. RLM showed the highest $\mathrm{CL}_{\text {int }}$ and DLM had the lowest $\mathrm{CL}_{\text {int }}$.

Data from cDNA-expressed CYP450s showed that M2 formation in HLM was mainly catalyzed by CYP2C9 and CYP2C19, and involvement of CYP3A4 in M2 formation was minor. In consistent with the previous reports [9, 10], M7 formation from DPT was mainly mediated by CYP3A4. Experiments in cDNA-expressed CYP450s showed that CYP3A4 may catalyze M2 metabolism to M1, and M1 formation from M7 was mainly attributed to CYP2C9 and CYP2C19, with minor contribution of CYP3A4 (data not shown).

The contributions of the three CYP450 isoforms to M2 formation in HLM were evaluated by specific chemical inhibitors and cDNA-expressed CYP450s. In contrary to our expectation, although cDNA-expressed CYP2C19 showed high catalytic activity of M2 formation, ticlopidine (CYP2C19 inhibitor) did not show inhibition on M2 formation in HLM. Low percent contribution of CYP2C19 to M2 formation in HLM may be partly due to the low content of CYP2C19 in HLM [16]. In consistent 
with findings in chemical inhibition, the rank of percent contribution to M2 formation in HLM was CYP2C9>CYP2C19>CYP3A4. It was noticed that the estimated contribution of CYP2C9 was over 100\%. RAF method was based on the following assumptions [21]: (1) The diagnostic substrate must be absolutely CYP450 isoform-selective. (2) The metabolic rate of diagnostic and tested substrates must increase linearly with the amounts of enzyme, without influence of non-specific microsomal binding. (3) Factors which influence metabolic rates in HLM and recombinant CYP450 systems must be equal across different substrates. However, these assumptions may not be fully met under all circumstances. Firstly, although the marker reaction of substrate is assumed to be specific for one CYP isoform, other CYP isoforms are involved in the metabolic reaction. For example, diclofenac 4'-hydroxylation was also catalyzed by CYP1A2, CYP3A4 and other members of CYP2C subfamily (CYP2C19, CYP2C8 and CYP2C18) apart from CYP2C9 [22, 23]. A report [24] showed that CYP3A4 was involved in omeprazole 5-hydroxylation (considered as specific reaction of CYP2C19), although the contribution of CYP3A4 was smaller than that of CYP2C19. Secondly, if non-specific microsomal binding of DPT existed in HLM, the CL $\mathrm{LLM}_{\mathrm{HL}}$ in Eq. 2 may be underestimated, leading to the overestimation of contribution. Thirdly, substrates may respond differently to the alteration of reaction system (in HLM or recombinant CYPs). An example of this was seen for CYP2B6 activity, which was highly enhanced by cytochrome b5 with the diagnostic substrate 7-ethoxy-4-trifluoromethylcoumarin, but not with the test substrates cyclophosphamide and ifosfamide [21]. Ratio of $\mathrm{CL}_{\text {int }}$ in HLM to that in 
Baculosomes (RAF value) of tolbutamide was reported to be $19.2 \mathrm{pmol} / \mathrm{mg}$, much lower than that of diclofenac $(73.7 \mathrm{pmol} / \mathrm{mg})$ [25], indicating that RAF value was substrate-dependent. All these reasons may result in overestimation of CYP2C9 contribution to M2 formation, which needed further investigation.

Several papers have investigated into the metabolism of DPT. Studies using recombinant CYP3A4 in E.coli focused on the biotransformation from DPT to M7 $[9,10]$. Lee SK et al identifited meatbolites of DPT in HLM and RLM and screened the responsible CYP450s in a qualitative way [8]. All the above authors put importance on the contribution of CYP3A4. In the present work, we first studied metabolism of DPT in a quantitative way and compared the contribution of the CYP450s. In contrast to previous speculation, although CYP3A4 resulted in formation of the most metabolite type, it didn't make an important contribution to total clearance of DPT.

In summary, seven metabolites of DPT in the liver microsomal incubations of human, monkey, rat, mouse and dog were identified. M2 was major metabolite in all five species. Large differences in the intrinsic clearance of M2 formation were found among species, the rank order of intrinsic clearance of M2 formation was RLM > CyLM > MLM > HLM> DLM. In HLM, M2 formation was mainly mediated by CYP2C9. 


\section{Acknowledgements}

This work was supported by the National Science foundation of China (No. 81473273, No. 81373482 and No. 81573490), the Fundamental Research Funds for the Central Universities (2015PT042, ZD2014YX0026 and PT2014YX0057) and the Priority Academic Program Development of Jiangsu Higher Education Institutions.

\section{References}

[1] Koulman A, Bos R, Medarde M, Pras N, Quax WJ. A fast and simple GC MS method for lignan profiling in Anthriscus sylvestris and biosynthetically related plant species. Planta Med. 2001;67:858-62.

[2] Wong SK, Tsui SK, Kwan SY, Su XL, Lin RC. Identification and characterization of Podophyllum emodi by API-LC/MS/MS. J Mass Spectrom. 2000;35:1246-51.

[3] Khaled M, Jiang ZZ, Zhang LY. Deoxypodophyllotoxin: a promising therapeutic agent from herbal medicine. J Ethnopharmacol. 2013;149:24-34.

[4] Guerram M, Jiang ZZ, Sun L, Zhu X, Zhang LY. Antineoplastic effects of deoxypodophyllotoxin, a potent cytotoxic agent of plant origin, on glioblastoma U-87 MG and SF126 cells. Pharmacol Rep. 2015;67:245-52.

[5] Jin M, Moon TC, Quan Z, Lee E, Kim YK, Yang JH, et al. The naturally occurring flavolignan, deoxypodophyllotoxin, inhibits lipopolysaccharide-induced iNOS expression through the NF-kappaB activation in RAW264.7 macrophage cells. Biol Pharm Bull. 2008;31:1312-5.

[6] Wang YR, Xu Y, Jiang ZZ, Guerram M, Wang B, Zhu X, et al. Deoxypodophyllotoxin induces G2/M cell cycle arrest and apoptosis in SGC-7901 cells and inhibits tumor growth in vivo. Molecules. 2015;20:1661-75.

[7] Yang Y, Chen Y, Zhong ZY, Zhang J, Li F, Jia LL, et al. Validated LC-MS/MS assay for quantitative determination of deoxypodophyllotoxin in rat plasma and its application in pharmacokinetic study. J Pharm Biomed Anal. 2014;88:410-5. 
[8] Lee SK, Jun IH, Yoo HH, Kim JH, Seo YM, Kang MJ, et al. Characterization of in vitro metabolites of deoxypodophyllotoxin in human and rat liver microsomes using liquid chromatography/tandem mass spectrometry. Rapid Commun Mass Spectrom. 2008;22:52-8.

[9] Vasilev NP, Julsing MK, Koulman A, Clarkson C, Woerdenbag HJ, Ionkova I, et al. Bioconversion of deoxypodophyllotoxin into epipodophyllotoxin in E. coli using human cytochrome P450 3A4. J Biotechnol. 2006;126:383-93.

[10] Julsing MK, Vasilev NP, Schneidman-Duhovny D, Muntendam R, Woerdenbag HJ, Quax WJ, et al. Metabolic stereoselectivity of cytochrome P450 3A4 towards deoxypodophyllotoxin: In silico predictions and experimental validation. Eur $\mathrm{J}$ Med Chem. 2008;43:1171-9.

[11] Abass K, Reponen P, Mattila S, Pelkonen O. Metabolism of carbosulfan. I. Species differences in the in vitro biotransformation by mammalian hepatic microsomes including human. Chem Biol Interact. 2009;181:210-9.

[12] Xie SS, Hu N, Jing XY, Liu XD, Xie L, Wang GJ, et al. Effect of Huang-Lian-Jie-Du-Decoction on pharmacokinetics of verapamil in rats. J Pharm Pharmacol. 2010;62:440-7.

[13] Faeste CK, Ivanova L, Uhlig S. In Vitro Metabolism of the Mycotoxin Enniatin B in Different Species and Cytochrome P450 Enzyme Phenotyping by Chemical Inhibitors. Drug Metab Dispos. 2011;39:1768-76.

[14] Khojasteh SC, Prabhu S, Kenny JR, Halladay JS, Lu AY. Chemical inhibitors of cytochrome P450 isoforms in human liver microsomes: a re-evaluation of P450 isoform selectivity. Eur J Drug Metab Pharmacokinet. 2011;36:1-16.

[15] Nakajima M, Nakamura S, Tokudome S, Shimada N, Yamazaki H, Yokoi T. Azelastine N-demethylation by cytochrome P-450 (CYP)3A4, CYP2D6, and CYP1A2 in human liver microsomes: evaluation of approach to predict the contribution of multiple CYPs. Drug Metab Dispos. 1999;27:1381-91.

[16] Liu X, Hu L, Ge G, Yang B, Ning J, Sun S, et al. Quantitative analysis of cytochrome $\mathrm{P} 450$ isoforms in human liver microsomes by the combination of proteomics and chemical probe-based assay. Proteomics. 2014;14:1943-51.

[17] Kaphalia L, Kaphalia BS, Kumar S, Kanz MF, Treinen-Moslen M. Efficient high performance liquid chromatograph/ultraviolet method for determination of diclofenac and 4'-hydroxydiclofenac in rat serum. J Chromatogr B Analyt Technol Biomed Life Sci. 2006;830:231-7.

[18] Yamada S, Shiohira H, Yasui-Furukori N, Tateishi T, Akamine Y, Uno T. The (R)-omeprazole hydroxylation index reflects CYP2C19 activity in healthy Japanese volunteers. Eur J Clin Pharmacol. 2013;69:1423-8.

[19] Fayer JL, Petullo DM, Ring BJ, Wrighton SA, Ruterbories KJ. A novel testosterone 6 beta-hydroxylase activity assay for the study of CYP3A-mediated metabolism, inhibition, and induction in vitro. J Pharmacol Toxicol Methods. 2001;46:117-23.

[20] Kajita J, Fuse E, Kuwabara T, Kobayashi H. The Contribution of Cytochrome P450 to the Metabolism of Tegafur in Human Liver. Drug Metab Pharmacokinet. 2003;18:303-9. 
[21] Roy P, Yu LJ, Crespi CL, Waxman DJ. Development of a substrate-activity based approach to identify the major human liver P-450 catalysts of cyclophosphamide and ifosfamide activation based on cDNA-expressed activities and liver microsomal P-450 profiles. Drug Metab Dispos. 1999;27:655-66.

[22] Ohyama K, Murayama N, Shimizu M, Yamazaki H. Drug interactions of diclofenac and its oxidative metabolite with human liver microsomal cytochrome P450 1A2-dependent drug oxidation. Xenobiotica. 2014;44:10-6.

[23] Mancy A, Antignac M, Minoletti C, Dijols S, Mouries V, Duong NT, et al. Diclofenac and its derivatives as tools for studying human cytochromes P450 active sites: particular efficiency and regioselectivity of P450 2Cs. Biochemistry. 1999;38:14264-70.

[24] Karam WG, Goldstein JA, Lasker JM, Ghanayem BI. Human CYP2C19 is a major omeprazole 5-hydroxylase, as demonstrated with recombinant cytochrome P450 enzymes. Drug Metab Dispos. 1996;24:1081-7.

[25] Kumar V, Rock DA, Warren CJ, Tracy TS, Wahlstrom JL. Enzyme source effects on CYP2C9 kinetics and inhibition. Drug Metab Dispos. 2006;34:1903-8. 
Table 1 Kinetic parameters of M2 formation in microsomes from five species

\begin{tabular}{cccccc}
\hline Parameters & Human & Monkey & Rat & Mouse & Dog \\
\hline $\mathrm{K}_{\mathrm{m} 1}(\mu \mathrm{M})$ & 0.24 & 0.08 & 0.05 & 1.81 & 0.09 \\
$\mathrm{~V}_{\max 1}(\mathrm{nmol} / \mathrm{min} / \mathrm{mg})$ & 0.32 & 0.40 & 4.93 & 3.43 & 0.03 \\
$\mathrm{CL}_{\text {int1 }}(\mathrm{mL} / \mathrm{min} / \mathrm{mg})$ & 1.38 & 4.90 & 94.8 & 1.89 & 0.30 \\
$\mathrm{~K}_{\mathrm{m} 2}(\mu \mathrm{M})$ & & 2.26 & & & 9.47 \\
$\mathrm{~V}_{\max 2}(\mathrm{nmol} / \mathrm{min} / \mathrm{mg})$ & & 1.02 & & & 0.10 \\
$\mathrm{CL}_{\text {int2 }}(\mathrm{mL} / \mathrm{min} / \mathrm{mg})$ & & 0.45 & & & 0.01 \\
$\mathrm{CL}_{\text {int,all }}(\mathrm{mL} / \mathrm{min} / \mathrm{mg})$ & 1.38 & 5.35 & 94.8 & 1.89 & 0.31 \\
\hline
\end{tabular}

$\mathrm{CL}_{\text {int } \mathrm{i}}$ was calculated as $\mathrm{V}_{\max \mathrm{i}} / \mathrm{K}_{\mathrm{mi}}$ and $C \mathrm{~L}_{\text {int,all }}$ was calculated as the addition of $\mathrm{CL}_{\text {int } 1}$ and $\mathrm{CL}_{\text {int } 2}$

Table 2 Contributions of CYP450 isoforms to M2 formation

\begin{tabular}{|c|c|c|c|}
\hline & CYP2C9 & CYP2C19 & CYP3A4 \\
\hline \multirow[t]{2}{*}{$\mathrm{CL}_{\text {rec-CYP450s }}(\mathrm{mL} / \mathrm{min} / \mathrm{pmol})$} & 0.098 & 0.069 & 0.002 \\
\hline & \multicolumn{3}{|c|}{ Method 1} \\
\hline $\mathrm{A}_{\mathrm{s}}(\mathrm{pmol} / \mathrm{mg})$ & 9.23 & 2.92 & 48.3 \\
\hline $\mathrm{CL}_{\mathrm{CYP} 450 \mathrm{~s}}(\mathrm{~mL} / \mathrm{min} / \mathrm{mg})$ & 0.91 & 0.20 & 0.08 \\
\hline \multirow[t]{2}{*}{ Contribution (\%) } & 65.7 & 14.6 & 5.6 \\
\hline & \multicolumn{3}{|c|}{ Method 2} \\
\hline $\mathrm{A}_{\text {rec-CYP450s }}(\mathrm{pmol} / \mathrm{min} / \mathrm{pmol})$ & 7.20 & 3.25 & 14.0 \\
\hline $\mathrm{A}_{\mathrm{HLM}}(\mathrm{nmol} / \mathrm{min} / \mathrm{mg})$ & 19.8 & 3.60 & 57.1 \\
\hline $\mathrm{RAF}_{\mathrm{CYP} 450 \mathrm{~s}}(\mathrm{pmol} / \mathrm{mg})$ & 27.5 & 11.1 & 40.8 \\
\hline $\mathrm{CL}_{\mathrm{CYP} 450 \mathrm{~s}}(\mathrm{~mL} / \mathrm{min} / \mathrm{mg})$ & 2.70 & 0.77 & 0.07 \\
\hline Contribution (\%) & 195.6 & 55.4 & 4.7 \\
\hline
\end{tabular}

$\mathrm{CL}_{\text {rec-CYP450s }}$ represents the intrinsic clearance $\left(\mathrm{V}_{\max } / \mathrm{K}_{\mathrm{m}}\right)$ of M2 formation in specific recombinant $\mathrm{CYP}$ isoforms. $\mathrm{A}_{\mathrm{s}}$ represents protein content of each $\mathrm{CYP}$ isoform in human liver microsomes from Liu $\mathrm{X}$ et al's work [22]. $\mathrm{CL}_{\mathrm{CYP} 450 \mathrm{~s}}$ represents intrinsic clearance of M2 formation via specific CYP450 isoform in HLM. $\mathrm{A}_{\text {rec-CYP450s }}$ and $\mathrm{A}_{\mathrm{HLM}}$ represent hydroxylation activities of marker substrates for specific CYP isoforms in recombinant CYPs and in human liver microsomes, respectively. 
Figure captions

Fig. 1. Chemical structures of deoxypodophyllotoxin (DPT) and seven metabolites $\mathrm{R} 1=\mathrm{R} 2=\mathrm{H} \quad$ deoxypodophyllotoxin $\mathrm{R} 1=\mathrm{H}, \mathrm{R} 2=\mathrm{OH} \quad \mathrm{M} 7$

$\mathrm{R} 3=\mathrm{R} 4=\mathrm{H} \quad \mathrm{M} 2$

$\mathrm{R} 3=\mathrm{H}, \mathrm{R} 4=\mathrm{OH} \quad \mathrm{M} 1$

$\mathrm{R} 5=\mathrm{R} 6=\mathrm{H} \quad \mathrm{M} 5 / 6$

$\mathrm{R} 5=\mathrm{H}, \mathrm{R} 6=\mathrm{OH} \quad \mathrm{M} 3 / 4$

Fig. 2. Representative Chromatograms of standards of DPT and its metabolites $(0.5$ $\mu \mathrm{M})(\mathrm{A})$ and Chromatograms of DPT and its metabolites in microsomes from human (B), cynomolgus monkey (C), rat (D), mouse (E) and beagle dog (F) following incubation for $30 \mathrm{~min}$. Numbers on the left of each channel represent corresponding peak height.

Fig. 3. Elimination of DPT and formation of M1, M2, M7 in HLM (A), CyLM (B), RLM (C), MLM (D) and DLM (E).

Fig. 4. Kinetic profiles for formation of M2 in HLM (A), CyLM (B), RLM(C), MLM (D) and DLM (E). Insets right below are Eadie-Hofstee plots for each of the profiles. Data are reported as mean \pm SD. Incubations were carried out across a 0.0196 to $12.57 \mu \mathrm{M}$ substrate concentration range.

Fig. 5. Effects of sulfaphenazole, ticlopidine and ketoconazole on M2 formation in HLM. Data are reported as mean \pm SD.

Fig. 6. M2 formation in cDNA-expressed recombinant CYP450s (A), kinetic profiles for M2 formation in recombinant CYP2C9 (B) and CYP2C19 (C) and CYP3A4 (D). 
Figures

Figure 1
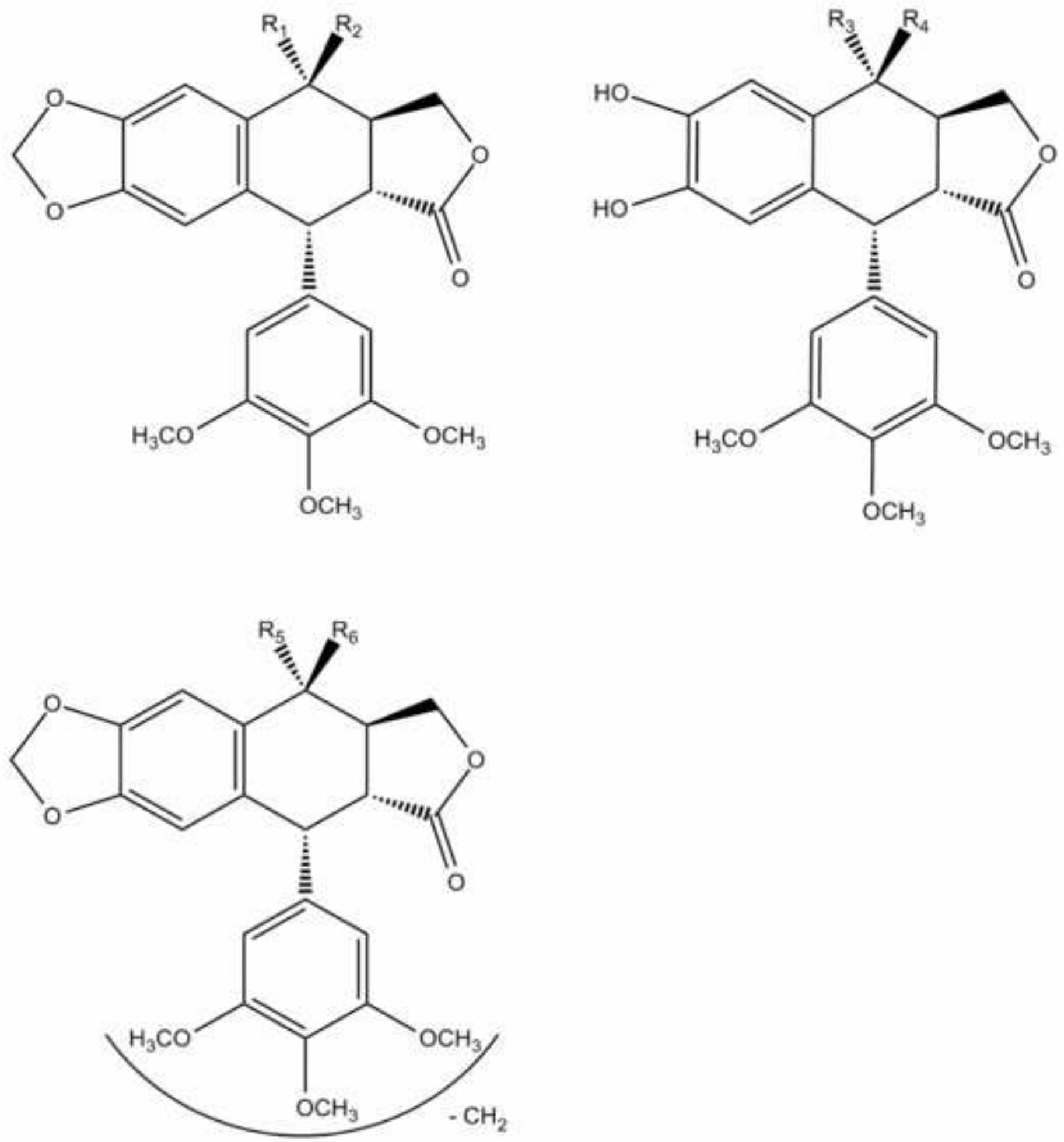
(A)

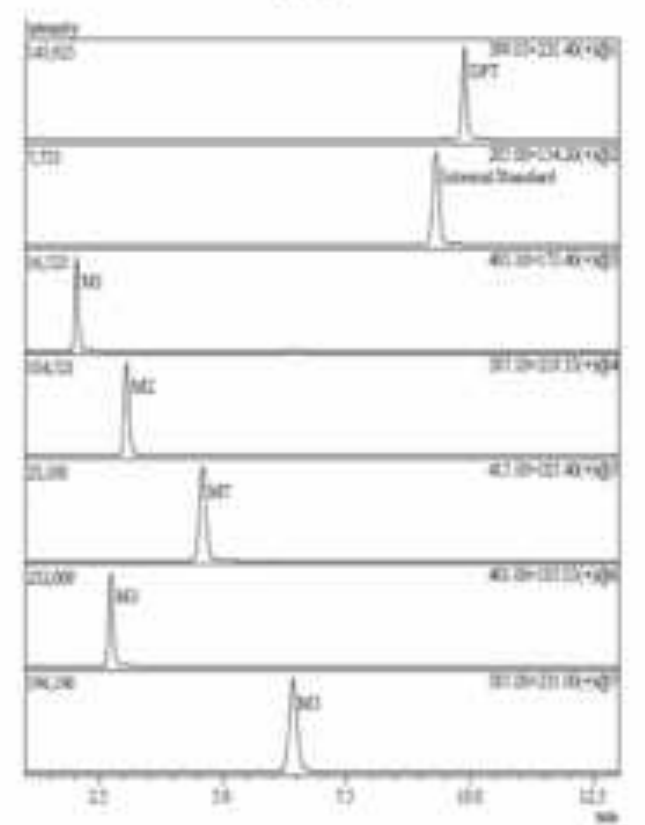

(D)

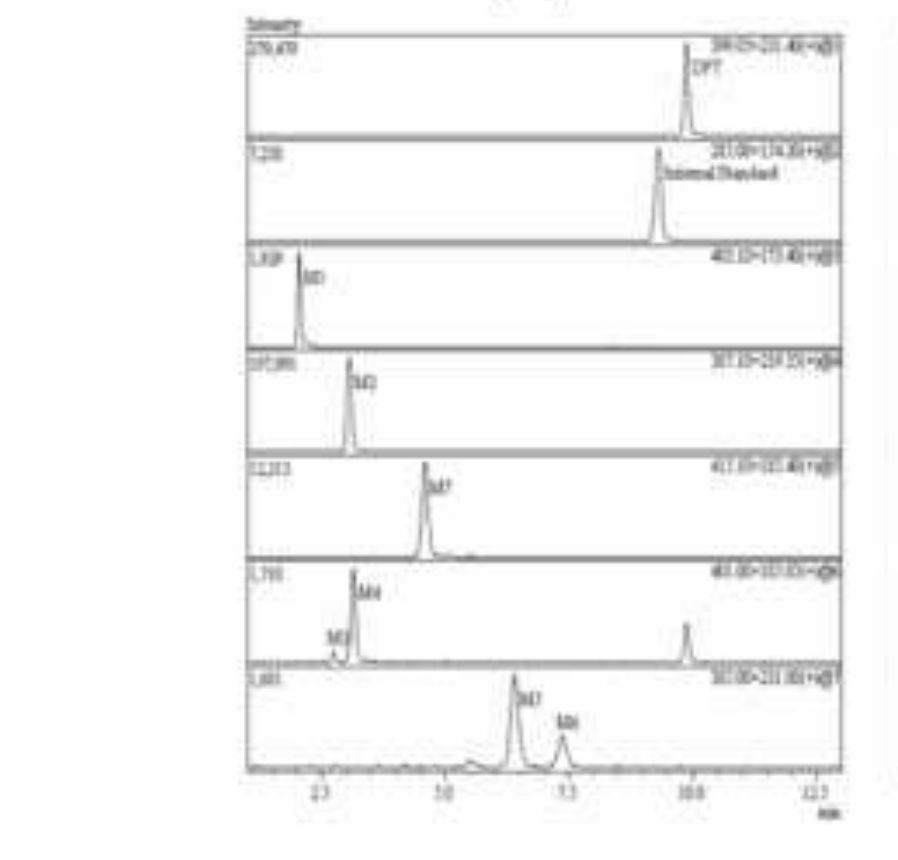

(B)

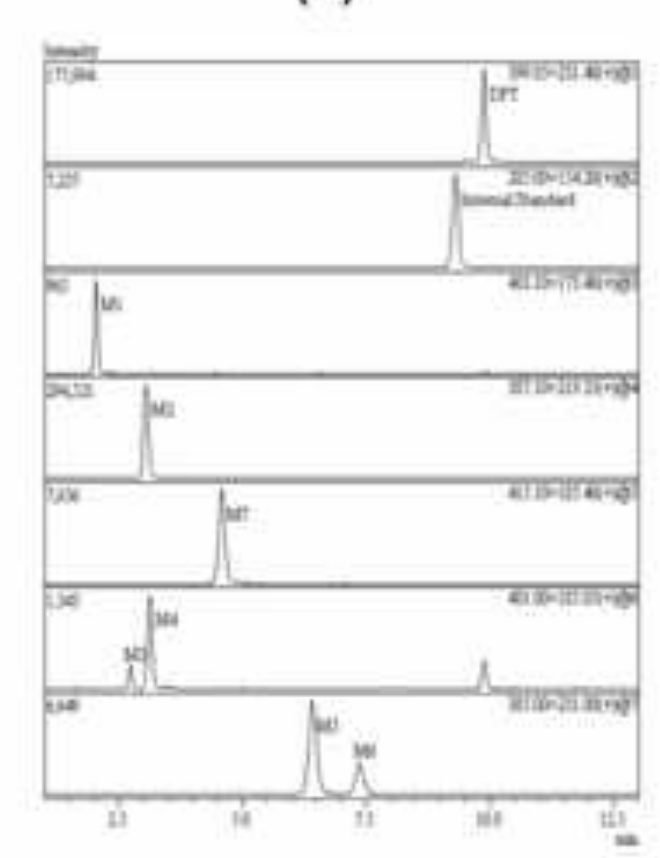

(E)

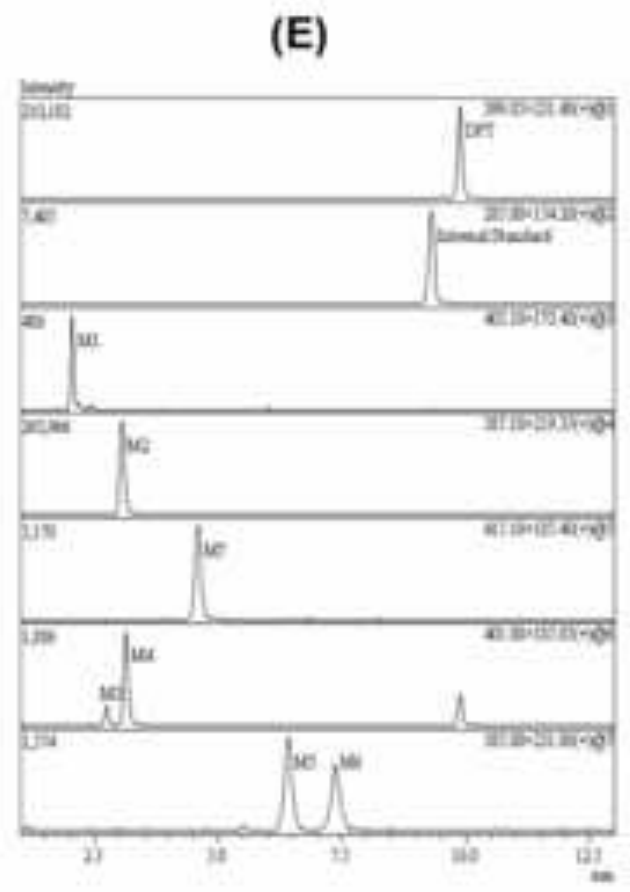

(C)

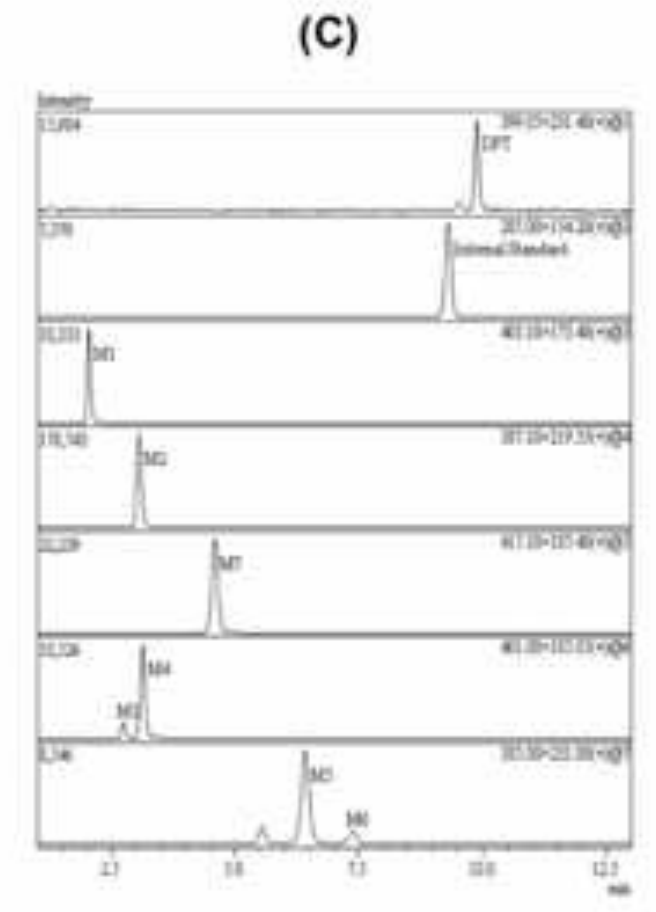

(F)

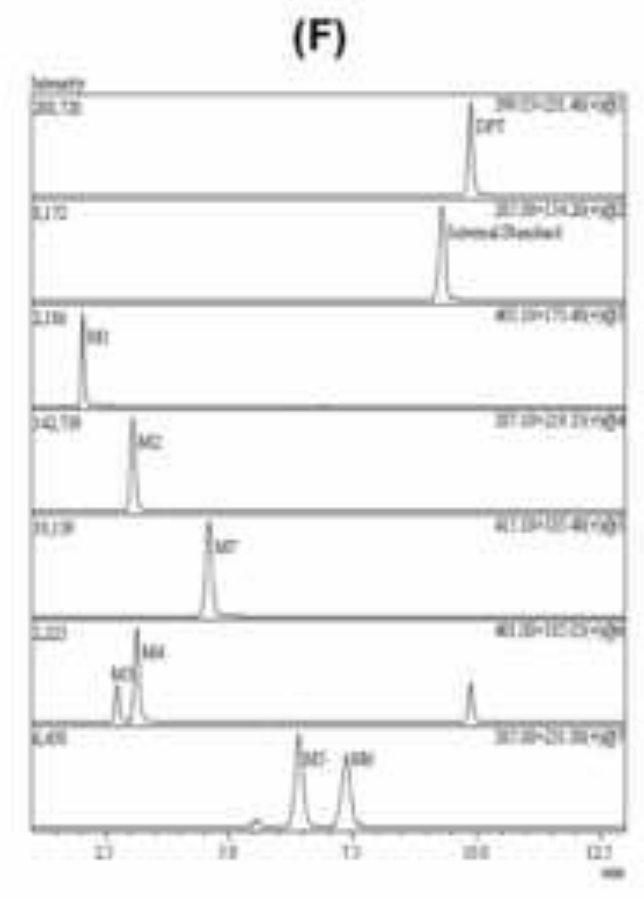



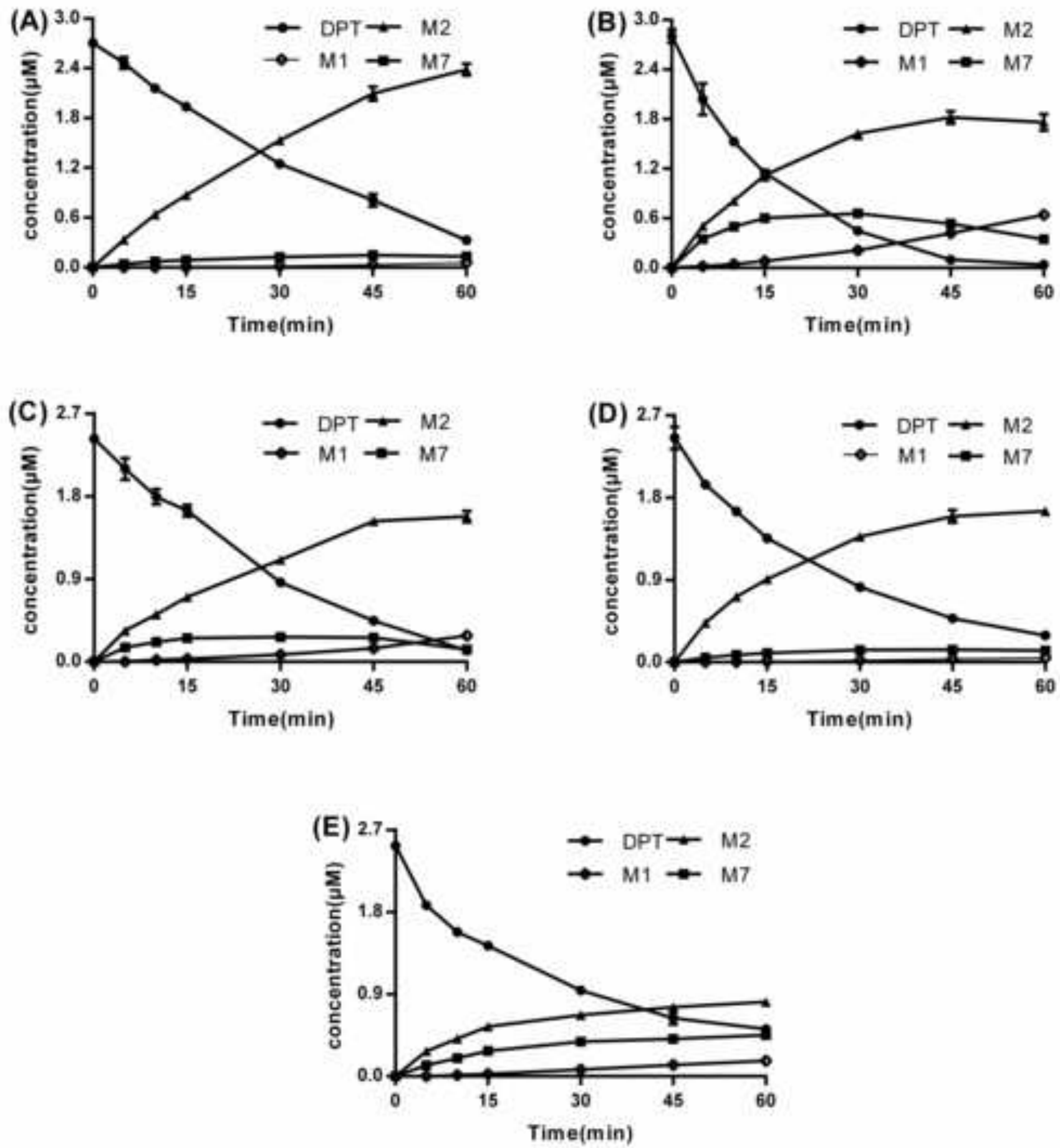

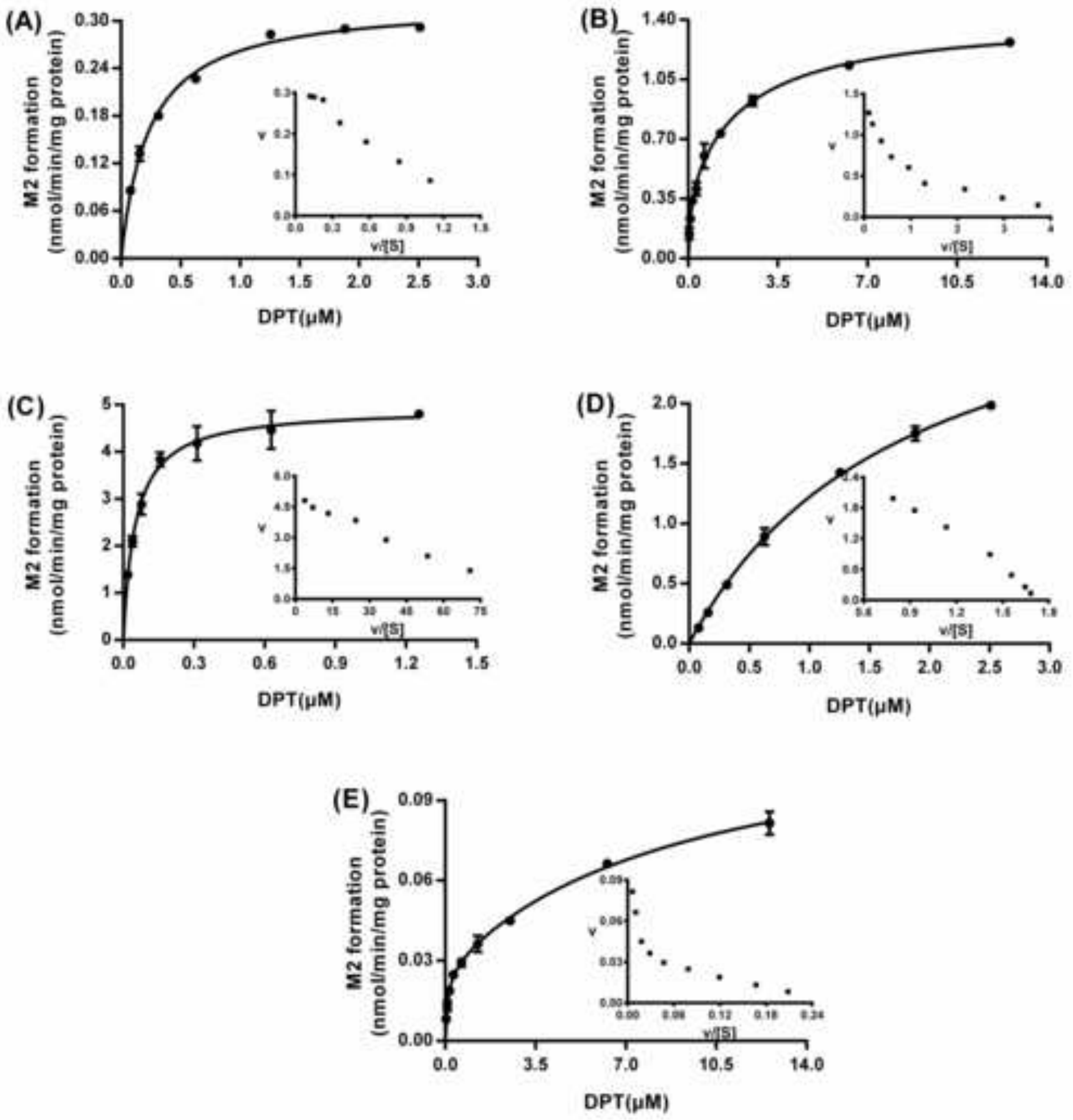


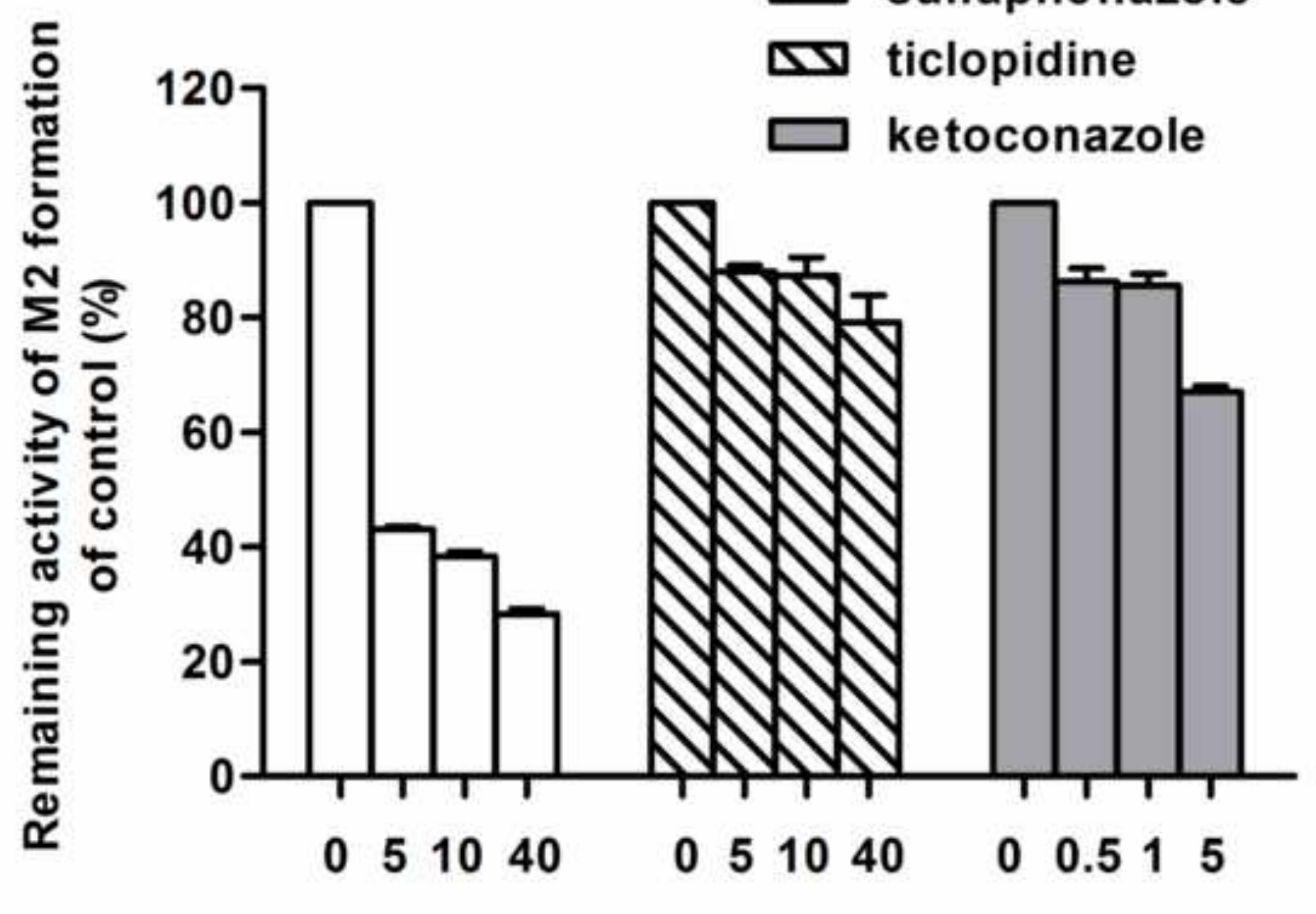

Concentration of chemical inhibitors $(\mu \mathrm{M})$ 

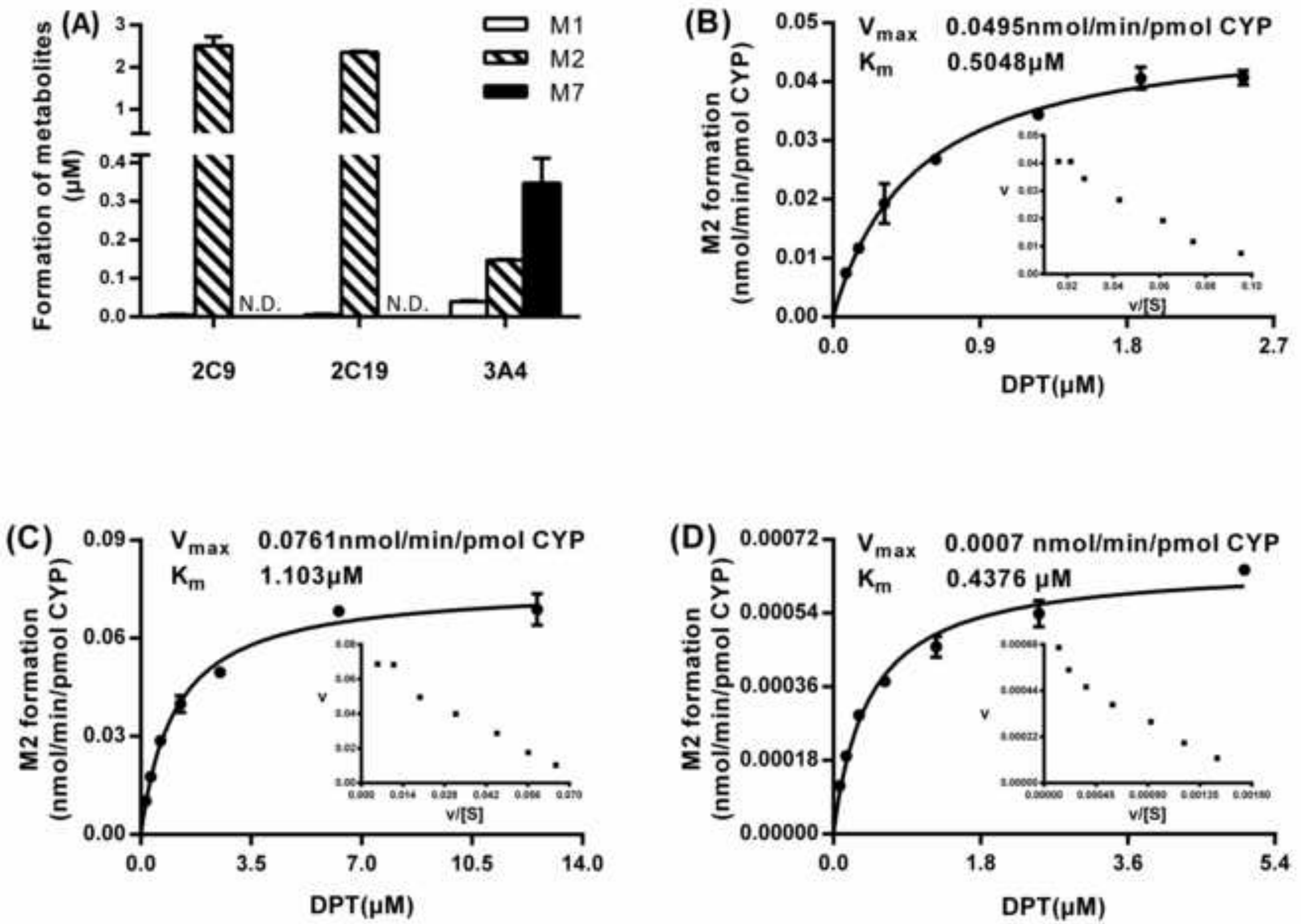